\title{
TWISTOR SPACES FOR REAL FOUR-DIMENSIONAL LORENTZIAN MANIFOLDS
}

\author{
YOSHINORI MACHIDA AND HAJIME SATO
}

\section{Introduction}

It is R. Penrose who constructed the twistor theory which gives a correspondence between complex space-times and 3-dimensional complex manifolds called twistor spaces. He and his colleagues investigated conformally invariant equations (e.g. massless field equations, self-dual Yang-Mills equations) on the space-time by transforming them into objects in complex analytical geometry. See e.g. Penrose-Ward [P-W] or Ward-Wells [W-W]. After that, Atiyah-Hitchin-Singer ([A-H-S], cf. [Fr]) constructed the twistor spaces corresponding to real 4-dimensional Riemannian manifolds. Their construction as well as that of Penrose is mainly effective under the condition of the self-duality. In this paper we will construct twistor spaces more geometrically from real 4-dimensional Lorentzian manifolds under a suitable curvature condition.

For a Lorentzian manifold, we have $*^{2}=-$ id for Hodge's star-operator * and the space of 2 -forms has no canonical real decomposition. The set of all Lorentz orthogonal complex structures on $\mathbf{R}_{1}^{4}$ is isomorphic to $S O_{+}(3,1) / U(1)$ and of 5 -dimension. It is different from the Riemannian case. After the work of $[\mathrm{A}-\mathrm{H}-\mathrm{S}]$, Berard Bergery and Ochiai [BB-O] generalized the theory from the viewpoint of $G$-structures. Since each fibre of their twistor space has to be a G-invariant subset of all complex structures, we need a different construction of a twistor space.

As the underlying space of the twistor space of a 4-dimensional Lorentzian manifold $M$, we take the space $P$ of all future-pointing null directions on $M$. This fits in with the original idea of Penrose and it will have physical applications. The space $P$ is a fibre bundle over $M$ with fibre $S^{2}$. By choosing a future-pointing timelike vector field $T$ on $M$, we can naturally define an almost complex structure $J=J_{T}$ on $P$.

Receivd May 26, 1993. 
The point $x$ and a timelike unit tangent vector $T$ at $x$ form a model of the instantaneous observer in the Lorentzian world $M$. The vector indicates the direction of the observer's individual time. The subspace in $M$ Lorentz orthogonal to this vector is a model of the 3-dimensional physical space of the instantaneous observer. The physical spaces of two different instantaneous observers are different, even if the observers are located at the same point of $M$. See Manin [Ma].

Our main result is to state the integrability condition of the almost complex structure $J$ on $P$ by the vanishing of certain parts of an irreducible $S O(3)$ decomposition of the curvature tensor $R$ of the 4 -dimensional Lorentzian manifold $M$.

This paper is organized as follows: In $\S 1$, we define an almost complex structure $J$ on the set $P$ of all future-pointing null directions on $M$, and we state the main theorem. We prove the theorem dividing into several steps in the next sections. In $\S 2$ and $\S 3$, we give a curvature condition of the integrability. We transfer the integrability condition to that of the linear frame bundle. We obtain a curvature condition which is the vanishing of two components of an irreducible $S O(3)$-decomposition. The irreducible $S O(3)$-decomposition of the curvature tensor is explained in Remark $A$ of $\S 5$. In $\S 4$, we give global examples with integrable twistor spaces. Any Robertson-Walker space-time is conformally flat and the flow of the perfect fluid on it defines an integrable twistor space. In Remark B of $\S 5$, we explain the relation of our result and that of [BB-O]. In Remark $\mathrm{C}$, we remark a relation to the normal $\mathrm{CR}$-structures of the tangent sphere bundles of 3 -dimensional manifolds ([S-Y]) and we will discuss a relation of our result to the twistor of the real 4-dimensional Riemannian manifold made by changing a real time to the imaginary time.

\section{§1. Almost complex structure of $P$}

1. Let $\left(M_{1}^{4}, g\right)$ be a 4-dimensional oriented and time-oriented connected Lorentzian manifold. Let $P$ be the set of all future-pointing null directions:

$$
P=\mathrm{Null}_{+}(M)=\{[v] \mid g(v, v)=0, v(\in T(M)) \text { is future-pointing }\},
$$

where $[v]$ is the equivalence class defined by the relation $v \sim \lambda v$. Then $P$ is a 6-dimensional manifold and is the total space of a fibre bundle over $M$ with fibre $S^{2}$. We define an almost complex structure on $P$ and study its integrability. When the structure is integrable, we call the complex 3 -dimensional manifold $P$ the integrable twistor space, or simply, the twistor space of the real 4-dimensional Lorentzian manifold $M$. 
On the integrable twistor space $P$, each fibre $S^{2}$ over $M$ becomes biholomorphic to $P^{1}(\mathbf{C})$, and the normal bundle to it is isomorphic to $H \oplus H$. Here $H$ is the hyperplane-section line bundle on $P^{1}(\mathbf{C})$.

The linear frame bundle over $M$ is reduced to a principal bundle $Q$ with structure group $S O_{+}(3,1)$. The space $P$ is a fibre bundle

$$
Q \times \times_{S O_{+}(3,1)} S^{2}
$$

with fibre

$$
S O_{+}(3,1) / H \cong S^{2}
$$

associated to $Q$. Let

$$
\pi: P \rightarrow M
$$

be the canonical projection. We will define an almost complex structure

$$
J: T P \rightarrow T P .
$$

We have the horizontal lift and the horizontal space of $M$ over $P$ associated to $Q$. Let $T^{v}$ be the vertical subbundle of $M$ and let $T^{h}$ be the horizontal subbundle of $M$. Then we have

$$
T P=T^{v} \oplus T^{h}
$$

On 2-dimensional subbundle $T^{v}$, an almost complex structure is naturally defined. On $T^{h}$, the problem is how we define an almost complex structure.

Let $[n]$ be a point in $P$. We want to lift the Lorentz orthogonal complex structures on $T_{\pi[n]} M \cong \mathbf{R}_{1}^{4}$ to $T^{h}$ horizontally. The set $C$ of all Lorentz orthogonal complex structures is a subset of $\operatorname{End}\left(T_{\pi[n]} M\right)$ and is isomorphic to $S O_{+}(3,1) / U(1)$. Therefore, since $C$ is 5 -dimensional, we cannot canonically define an almost complex structure on $T P$. Compare with the Riemannian case where the set $C$ of all orthogonal complex structures is isomorphic to $S O(4) / U(2) \cong S^{2}$. Let $T$ be a future-pointing timelike vector field on $M$ such that $g(T, T)=-1$. We will define an almost complex structure $J_{T}$ associated to $T$. Let $\Pi_{t}$ be a timelike plane spanned by $T$ and $n$. Let $\Pi_{s}$ be a spacelike plane orthogonal to $\Pi_{t}$. Then there exists an oriented Lorentz orthonormal basis $\left(e_{1} ; e_{2}, e_{3}, e_{4} \in T_{\pi[n]} M\right)$ such that

$$
e_{1}(=T), e_{2} \in \Pi_{t} ; e_{3}, e_{4} \in \Pi_{s}
$$

and

$$
g\left(e_{1}, e_{1}\right)=-1, g\left(e_{2}, e_{2}\right)=g\left(e_{3}, e_{3}\right)=g\left(e_{4}, e_{4}\right)=1, g\left(e_{i}, e_{j}\right)=0(i \neq j) .
$$


A vector $e_{2}$ is uniquely determined under the condition that $g\left(T+e_{2}, n\right)=0$. If we define a transformation $j_{T}$ on $T_{\pi[n]} M$ such that

$$
j_{T}\left(e_{1}\right)=e_{2}, \quad j_{T}\left(e_{2}\right)=-e_{1} ; \quad j_{T}\left(e_{3}\right)=e_{4}, \quad j_{T}\left(e_{4}\right)=-e_{3},
$$

we define a Lorentz orthogonal complex structure on $T_{\pi[n]} M$. It is independent of the choice of $e_{3}, e_{4} \in \Pi_{s}$. Thus, when we choose $T$, we can canonically define a complex structure on $T_{\pi[n]} M$. If we define a transformation $J_{T}$ on $T_{[n]}^{h}$ such that

$$
J_{T}\left(e_{1}^{h}\right)=e_{2}^{h}, \quad J_{T}\left(e_{2}^{h}\right)=-e_{1}^{h} ; \quad J_{T}\left(e_{3}^{h}\right)=e_{4}^{h}, \quad J_{T}\left(e_{4}^{h}\right)=-e_{3}^{h},
$$

for the horizontal lifts $e_{1}^{h} ; e_{2}^{h}, e_{3}^{h}, e_{4}^{h}$, we define a complex structure on the horizontal space $T_{[n]}^{h}$ of $T_{[n]} P$. Therefore we can define an almost complex structure $J_{T}$ associated to $T$ on $T_{[n]} P$ and $T P$.

2. We will show the integrability of $J_{T}$ under the curvature conditions of $g$. In this section we state the main theorem. The proof will be given in the next sections. Let $x$ be a point in $M$. We regard the curvature tensor $R$ as a linear transformation on $\Lambda^{2}=\Lambda^{2} T_{x} M$ by

$$
g(R(X \wedge Y), Z \wedge W)=g(R(X, Y) Z, W)
$$

for $X, Y, Z, W \in T_{x} M$. Put

$$
R_{i j k l}=g\left(R\left(e_{i} \wedge e_{\jmath}\right), e_{k} \wedge e_{l}\right),
$$

for a $T$-Lorentz orthonormal basis $\left(e_{1} ; e_{2}, e_{3}, e_{4} \in T_{x} M\right)$. Here we mean a $T$-Lorentz orthonormal basis by a Lorentz orthonormal basis such that $e_{1}=T$. We write the dual basis of $T_{x}^{*} M$ for $\left(e_{\imath}\right)$ by the same letter $\left(e_{i}\right)$. We have the linear transformation

$$
R: \Lambda^{2} \rightarrow \Lambda^{2}
$$

by

$$
R\left(e_{i} \wedge e_{j}\right)=\frac{1}{2} \sum_{k, l} R_{i j k l} e_{k} \wedge e_{l}
$$

The class of six 2 -forms

$$
\tilde{\mathbf{e}}=\left(e_{1} \wedge e_{2}, e_{1} \wedge e_{3}, e_{1} \wedge e_{4} ; e_{3} \wedge e_{4}, e_{4} \wedge e_{2}, e_{2} \wedge e_{3}\right)
$$

is an orthonormal basis of $\Lambda^{2}$. The former three 2 -forms are of norm -1 and they span the timelike planes. The latter three 2 -forms are of norm 1 and they span the spacelike planes. We denote the subspaces of $\Lambda^{2}$ spanned by the former 
three 2 -forms and the latter three 2 -forms by $\Lambda_{t}^{2}$ and $\Lambda_{s}^{2}$ respectively. With respect to the orthonormal basis, Hodge's $*$-operator $*: \Lambda^{2} \rightarrow \Lambda^{2}$ is represented by $*=\left(\begin{array}{cc}O & -I \\ I & O\end{array}\right)$, where $I$ is the $3 \times 3$ unit matrix. The mapping $*$ transforms $\Lambda_{t}^{2}$ onto $\Lambda_{s}^{2}$ and $\Lambda_{s}^{2}$ onto $\Lambda_{t}^{2}$ and $*^{2}=-\mathrm{id}$. With respect to the mapping $*, \Lambda^{2}$ becomes a 3 -dimensional complex vector space.

Put $Q(\alpha, \beta)=g(R \alpha, \beta)$ for $\alpha, \beta \in \Lambda^{2}$. Then with respect to the orthonor. mal basis $\tilde{\mathbf{e}}$ of $\Lambda^{2}$, we can write

$$
\begin{aligned}
Q & =\left(\begin{array}{ll}
A & B \\
{ }^{t} B & C
\end{array}\right)=\left(\begin{array}{llllll}
R_{1212} & R_{1312} & R_{1412} & R_{3412} & R_{4212} & R_{2312} \\
R_{1213} & R_{1313} & R_{1413} & R_{3413} & R_{4213} & R_{2313} \\
R_{1214} & R_{1314} & R_{1414} & R_{3414} & R_{4214} & R_{2314} \\
R_{1234} & R_{1334} & R_{1434} & R_{3434} & R_{4234} & R_{2334} \\
R_{1242} & R_{1342} & R_{1442} & R_{3442} & R_{4242} & R_{2342} \\
R_{1223} & R_{1323} & R_{1423} & R_{3423} & R_{4223} & R_{2323}
\end{array}\right) \\
& =\left(\begin{array}{llllll}
\delta & d & e & x & p & q \\
d & \varepsilon & f & s & y & r \\
e & f & \zeta & t & u & z \\
x & s & t & \alpha & a & b \\
p & y & u & a & \beta & c \\
q & r & z & b & c & \gamma
\end{array}\right), \\
R & =\left(\begin{array}{ccc}
-A & -B \\
{ }^{t} B & C
\end{array}\right),
\end{aligned}
$$

where $A, B, C$ are $3 \times 3$ matrices and $\delta=R_{1212}, d=R_{1312}, e=R_{1412}, \ldots$ Then $A={ }^{t} A, C={ }^{t} C$, and $-A: \Lambda_{t}^{2} \rightarrow \Lambda_{t}^{2}, C: \Lambda_{s}^{2} \rightarrow \Lambda_{s}^{2}$ are self-adjoint linear transformations.

We can state the following main theorem:

Theorem. Suppose that $J_{T}$ is integrable on $P$. Then, with respect to any $T$-Lorentz orthonormal basis $\mathbf{e}=\left(e_{1}=T ; e_{2}, e_{3}, e_{4} \in T_{x} M\right)$ at any point $x \in M$, the following six relations hold:

$$
\left\{\begin{array} { l } 
{ p + s + 2 a = x - y + \delta - \varepsilon } \\
{ q + t + 2 b = z - x + \zeta - \delta } \\
{ r + u + 2 c = y - z + \varepsilon - \zeta }
\end{array} \quad \left\{\begin{array}{l}
p+s+2 d=-x+y-\alpha+\beta \\
q+t+2 e=-z+x-\gamma+\alpha \\
r+u+2 f=-y+z-\beta+\gamma
\end{array}\right.\right.
$$

Conversely, if this six relations hold for some $T$-Lorentz orthonormal basis $\mathbf{e}=\left(e_{1}=T\right.$; $\left.e_{2}, e_{3}, e_{4} \in T_{x} M\right)$ at any point $x \in M$, then $J_{T}$ is integrable on $P$. 
From the above six relations, it follows that

$$
a+b+c=e+d+f=-\frac{1}{2}(p+q+r+s+t+u) .
$$

By the first Bianchi's identity, $x+y+z=0$. Although $T$-Lorentz orthonormal basis at each point has an ambiguity of $S O(3)$, the six relations are invariant under the rotations by $S O(3)$.

3. The curvature tensor $R=\left(\begin{array}{cc}-A & -B \\ { }^{t} B & C\end{array}\right)$ is decomposed into the spaces $\mathscr{W}, \mathscr{L}, \mathcal{U}$, (see Besse $[\mathrm{Be}]$ ), where

$$
\begin{aligned}
& \mathscr{W}=\{R \mid * R=R *, \operatorname{tr} R=0\} \cdots \text { Ricci flat, } \\
& \mathscr{L}=\{R \mid * R=-R *\}, \\
& \mathcal{U}=\left\{R \mid R=c I_{6}, c: \text { constant, } I_{6}: 6 \times 6 \text { unit matrix }\right\} \cdots \text { constant curvature, } \\
& \mathcal{U}+\mathscr{W} \text { (i.e., the part of } \mathscr{L}=0) \cdots \text { Einstein }(* R=R * \text { ), } \\
& \mathcal{U}+\mathscr{L} \text { (i.e., the part of } \mathscr{W}=0) \cdots \text { conformally flat. }
\end{aligned}
$$

They are irreducible $S O_{+}(3,1)$-invariant subspaces of algebraic curvature tensor spaces $\mathscr{R}$ and $\operatorname{dim} \mathscr{W}=10, \operatorname{dim} \mathscr{L}=9, \operatorname{dim} \mathcal{U}=1$ (cf. Besse [Be]). Since

$$
\begin{aligned}
& \left.R \in \mathscr{W} \Leftrightarrow-A=C, B={ }^{t} B, \operatorname{tr} A=0 \text { (or } \operatorname{tr} C=0\right), \\
& R \in \mathscr{L} \Leftrightarrow A=C, B=-{ }^{t} B, \\
& R \in \mathcal{U} \Leftrightarrow-A=C=c I_{3}\left(R=c I_{6}\right),
\end{aligned}
$$

we have

$$
\begin{aligned}
R & =\left(\begin{array}{cc}
-A & -B \\
{ }^{t} B & C
\end{array}\right), \\
& =W(\in W)+L(\in \mathscr{W})+U(\in \mathcal{U}) \\
& =\left(\begin{array}{cc}
\frac{-A+C}{2}-\operatorname{tr} A \cdot I_{3} & \frac{-B-{ }^{t} B}{2} \\
\frac{B+{ }^{t} B}{2} & \frac{-A+C}{2}-\operatorname{tr} A \cdot I_{3}
\end{array}\right)+\left(\begin{array}{cc}
\frac{-A-C}{2} & \frac{-B+{ }^{t} B}{2} \\
\frac{-B+{ }^{t} B}{2} & \frac{A+C}{2}
\end{array}\right) \\
& +\operatorname{tr} A \cdot I_{6} .
\end{aligned}
$$

If the Lorentzian manifold $(M, g)$ is of restricted type, the integrability condition of $J_{T}$ becomes easier to describe. 
COROLlary. The necessary and sufficient condition that $J_{T}$ is integrable on $P$ is given as follows:

(1) If $R \in \mathcal{U}$, i.e., if $(M, g)$ is of constant curvature, then $J_{T}$ is integrable for any $T$ on $P$ and

$$
Q=\left(\begin{array}{cc}
-\alpha I & O \\
O & \alpha I
\end{array}\right),
$$

(2) If $R \in U+\mathscr{L}$, i.e., if $(M, g)$ is conformally flat, then the condition is that $Q$ is represented by the following form:

$$
Q=\left(\begin{array}{cccccc}
k & 0 & 0 & 0 & p & q \\
0 & k & 0 & -p & 0 & r \\
0 & 0 & k & -q & -r & 0 \\
0 & -p & -q & l & 0 & 0 \\
p & 0 & -r & 0 & l & 0 \\
q & r & 0 & 0 & 0 & l
\end{array}\right)
$$

(3) If $R \in \mathcal{U}+\mathscr{W}$, i.e., if $(M, g)$ is Einstein, then, for a form

$$
Q=\left(\begin{array}{cccccc}
-\alpha & -a & -b & x & p & q \\
-a & -\beta & -c & p & y & r \\
-b & -c & -\gamma & q & r & z \\
x & p & q & \alpha & a & b \\
p & y & r & a & \beta & c \\
q & r & z & b & c & \gamma
\end{array}\right)
$$

the condition is that

$$
\begin{gathered}
2 p=-\alpha+\beta, 2 q=-\gamma+\alpha, 2 r=-\beta+\gamma, \\
2 a=x-y, 2 b=z-x, 2 c=y-z, \\
(x+y+z=0) .
\end{gathered}
$$

Especially, if

$$
p=q=r=0
$$

then

$$
\alpha=\beta=\gamma
$$

and if

$$
a=b=c=0 \text {, }
$$


then

$$
x=y=z=0 .
$$

Note that, if $R \in W$, i.e., if $(M, g)$ is Ricci flat, then the condition in (3) is that

$$
\begin{gathered}
2 p=-\alpha+\beta, 2 q=-\gamma+\alpha, 2 r=-\beta+\gamma, \\
2 a=x-y, 2 b=z-x, 2 c=y-z, \\
\alpha+\beta+\gamma=0, \\
(x+y+z=0) .
\end{gathered}
$$

In $\S 4$, we will give examples with integrable twistor spaces.

\section{§2. Integrability of $J_{T}$}

1. We investigate the integrability of $J_{T}$ not on $P$ directly but on the linear frame bundle over $M$. Let $x$ be a point in $M$ and let $\mathbf{e}=\left(e_{1}=T ; e_{2}, e_{3}, e_{4}\right)$ be a $T$-Lorentz orthonormal basis on $T_{x} M$. Then $Q^{\prime}=\cup_{x \in M}\{\mathbf{e}\}$ is the principal $S O(1) \times S O(3) \cong S O(3)$ subbundle of $Q$ over $M$. We define a mapping

$$
\Phi: Q^{\prime} \rightarrow P
$$

by

$$
\Phi(\mathbf{e})=\left[e_{1}+e_{2}\right]
$$

Then $Q^{\prime}$ is a principal $S O(2)$ bundle over $P$. Similarly we define a mapping

$$
\Phi: Q \rightarrow P
$$

and $Q$ is a principal $H$ bundle over $P$. We have the following diagrams:

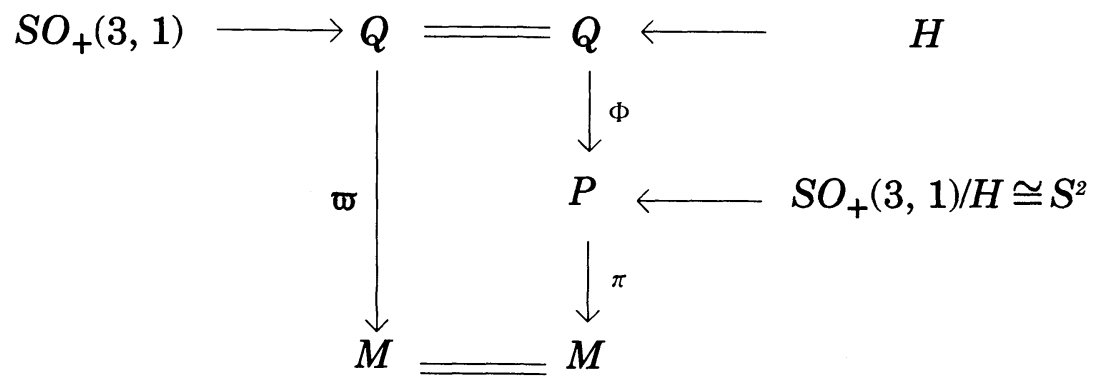

By a choice of $T$, we have the following reduced digrams: 


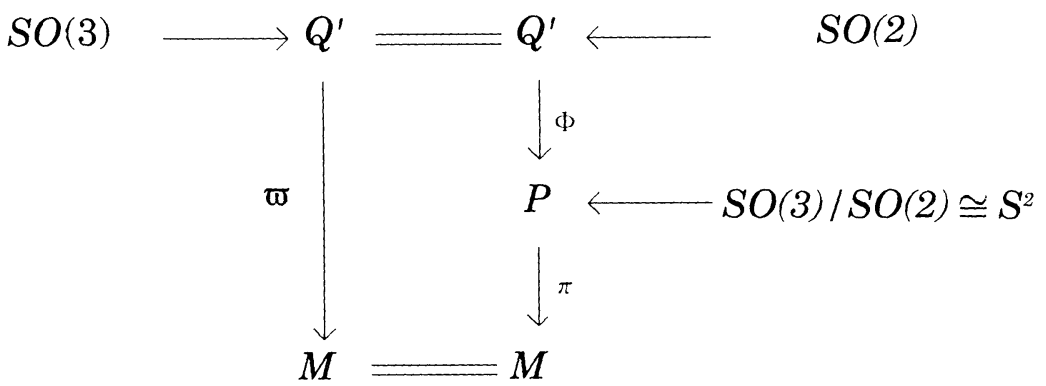

2. For a $T$-Lorentz orthonormal basis $\mathbf{e}=\left(e_{1}=T ; e_{2}, e_{3}, e_{4}\right)$ on $T_{x} M$. put $n=e_{1}+e_{2} \in T_{x} M$. The horizontal space $T_{[n]}^{h} \subset T_{[n]} P$ and the horizontal lifts $e_{1}^{h} ; e_{2}^{h}, e_{3}^{h}, e_{4}^{h} \in T_{[n]}^{h}$ were defined in $\$ 1$. Similarly we can define the horizontal space $T_{\mathbf{e}}^{H} \subset T_{\mathbf{e}} Q$ and the horizontal lifts $e_{1}^{H} ; e_{2}^{H}, e_{3}^{H}, e_{4}^{H} \in T_{\mathbf{e}}^{H}$ by $S O_{+}(3,1)-$ connection. Since the holonomy group of $M$ is not always $S O(3), S O_{+}(3,1)$ connection is not always reduced to $S O(3)$-connection on $Q^{\prime}$. Thus $T_{\mathrm{e}}^{H}$ is not always a subspace of $T_{\mathbf{e}}^{H} Q^{\prime}$. We can define a complex structure $\bar{J}_{T}$ on $T_{\mathbf{e}}^{H}$ by

$$
\bar{J}_{T}\left(e_{1}^{H}\right)=e_{2}^{H}, \bar{J}_{T}\left(e_{2}^{H}\right)=-e_{1}^{H}, \bar{J}_{T}\left(e_{3}^{H}\right)=e_{4}^{H}, \bar{J}_{T}\left(e_{4}^{H}\right)=-e_{3}^{H} .
$$

For $\mathbf{e}=\left(e_{1}=T ; e_{2}, e_{3}, e_{4}\right) \in Q^{\prime}$ and

$$
a=\left(\begin{array}{cccc}
1 & 0 & 0 & 0 \\
0 & 1 & 0 & 0 \\
0 & 0 & \\
0 & 0 & a^{\prime}
\end{array}\right) \in S O(3) \subset S O_{+}(3,1), \quad a^{\prime} \in S O(2),
$$

we have $R_{a} \mathbf{e}=\left(e_{1}=T ; e_{2}, e_{3} a, e_{4} a\right) \in Q^{\prime}$. On $T_{R_{a} \mathbf{e}}^{H}$ we have

$$
\bar{J}_{T}\left(e_{1}^{H}\right)=e_{2}^{H}, \bar{J}_{T}\left(e_{2}^{H}\right)=-e_{1}^{H} ; \bar{J}_{T}\left(\left(e_{3} a\right)^{H}\right)=\left(e_{4} a\right)^{H}, \bar{J}_{T}\left(\left(e_{4} a\right)^{H}\right)=-\left(e_{3} a\right)^{H}
$$

and easily get

$$
\bar{J}_{T}\left(e_{3}^{H}\right)=e_{4}^{H}, \quad \bar{J}_{T}\left(e_{4}^{H}\right)=-e_{3}^{H} \quad \text { for } \quad e_{3}, e_{4} \in T_{x} M
$$

LeMva. $\bar{J}_{T}$ is $S O(2)$ equivariant, that is,

$$
R_{a *} \bar{J}_{T}=\bar{J}_{T} R_{a *}
$$

Proof. It is sufficient to prove $R_{a *} \bar{J}_{T}\left(e_{i}^{H}\right)=\bar{J}_{T} R_{a *}\left(e_{i}^{H}\right)$ for $e_{i}^{H}, i=1,2,3,4$, of $T_{\mathbf{e}}^{H}$. Observe that, since we have $R_{a *} T_{\mathbf{e}}^{H}=T_{R_{a}}^{H}$, the horizontal space in $T Q$ at a 
point on $Q^{\prime}$ is $S O(2)$-invariant. In general we have $R_{a *}\left(v^{H}\right)=v^{H}$ for $v \in T_{x} M$, $a \in S O_{+}(3,1)$. Therefore

$$
R_{a *} \bar{J}_{T}\left(e_{1}^{H}\right)=R_{a *} e_{2}^{H}=e_{2}^{H}
$$

and

$$
\bar{J}_{T} R_{a *}\left(e_{1}^{H}\right)=\bar{J}_{T} e_{1}^{H}=e_{2}^{H} .
$$

Thus $R_{a *} \bar{J}_{T}\left(e_{1}^{H}\right)=\bar{J}_{T} R_{a *}\left(e_{1}^{H}\right)$. Similarly $R_{a *} \bar{J}_{T}\left(e_{i}^{H}\right)=\bar{J}_{T} R_{a *}\left(e_{i}^{H}\right), i=2,3,4$.

3. Let $E_{i j}$ be the matrix unit whose $(i, j)$-component is equal to 1 and the other components are 0 . For $S=\left(\begin{array}{cc}-1 & 0 \\ 0 & I_{3}\end{array}\right)$, let us put

$$
\begin{aligned}
S O(3,1) & =\left\{X \in G L(4, \mathbf{R}) \mid{ }^{t} X S X=S\right\}, \\
\mathcal{o}(3,1) & =\left\{X \in \operatorname{Mat}(4, \mathbf{R}) \mid{ }^{t} X S+S X=0\right\} .
\end{aligned}
$$

Then $\mathrm{o}(3,1)$ has the basis $\left(X_{1}, X_{2}, X_{3} ; Y_{1}, Y_{2}, Y_{3}\right)$ defined by

$$
\begin{aligned}
& X_{1}=\left(\begin{array}{llll}
0 & 1 & 0 & 0 \\
1 & 0 & 0 & 0 \\
0 & 0 & 0 & 0 \\
0 & 0 & 0 & 0
\end{array}\right)=E_{12}+E_{21}, \quad X_{2}=\left(\begin{array}{llll}
0 & 0 & 1 & 0 \\
0 & 0 & 0 & 0 \\
1 & 0 & 0 & 0 \\
0 & 0 & 0 & 0
\end{array}\right)=E_{13}+E_{31}, \\
& X_{3}=\left(\begin{array}{llll}
0 & 0 & 0 & 1 \\
0 & 0 & 0 & 0 \\
0 & 0 & 0 & 0 \\
1 & 0 & 0 & 0
\end{array}\right)=E_{14}+E_{41}, \quad Y_{1}=\left(\begin{array}{cccc}
0 & 0 & 0 & 0 \\
0 & 0 & 0 & 0 \\
0 & 0 & 0 & -1 \\
0 & 0 & 1 & 0
\end{array}\right)=-E_{34}+E_{43}, \\
& Y_{2}=\left(\begin{array}{llll}
0 & 0 & 0 & 0 \\
0 & 0 & 0 & -1 \\
0 & 0 & 0 & 0 \\
0 & 1 & 0 & 0
\end{array}\right)=-E_{24}+E_{42}, \quad Y_{3}=\left(\begin{array}{cccc}
0 & 0 & 0 & 0 \\
0 & 0 & -1 & 0 \\
0 & 1 & 0 & 0 \\
0 & 0 & 0 & 0
\end{array}\right)=-E_{23}+E_{32} .
\end{aligned}
$$

Obviously the subgroup

$$
\left\{a \in S O_{+}(3,1) \mid a=\left(\begin{array}{cccc}
1 & 0 & 0 & 0 \\
0 & & & \\
0 & & a^{\prime} \\
0 & &
\end{array}\right), a^{\prime} \in S O(3)\right\}
$$

is isomorphic to $S O(3)$. The corresponding Lie subalgebra of $\mathrm{o}(3,1)$ is isomorphic to $\mathrm{o}(3)$ and $Y_{1}, Y_{2}, Y_{3}$ are the basis on it. Similarly the subgroup 


$$
\left\{a \in S O_{+}(3,1) \mid a=\left(\begin{array}{llll}
1 & 0 & 0 & 0 \\
0 & 1 & 0 & 0 \\
0 & 0 & \\
0 & 0 & a^{\prime}
\end{array}\right), a^{\prime} \in S O(2)\right\}
$$

is isomorphic to $S O(2)$. The corresponding Lie subalgebra of $\mathrm{o}(3,1)$ is isomorphic to $\mathrm{o}(2)$ and $Y_{1}$ is the basis on it.

By the way, $\mathrm{o}(3,1)$ has the structure of a simple graded Lie algebra as follows. Put

$$
\begin{aligned}
g_{0} & =\operatorname{span}\left\{X_{1}, Y_{1}\right\}, \\
\mathfrak{g}_{1} & =\operatorname{span}\left\{V_{1}=X_{2}-Y_{3}, V_{2}=X_{3}-Y_{2}\right\}, \\
\mathfrak{g}_{-1} & =\operatorname{span}\left\{W_{1}=X_{2}+Y_{3}, W_{2}=X_{3}+Y_{2}\right\} .
\end{aligned}
$$

Then we have

$$
\begin{aligned}
& \mathfrak{o}(3,1)=\mathfrak{g}_{-1} \oplus \mathfrak{g}_{0} \oplus \mathfrak{g}_{1}, \\
& {\left[\mathfrak{g}_{i}, \mathfrak{g}_{j}\right] \subset \mathfrak{g}_{i+j} .}
\end{aligned}
$$

Furthermore, for the Lie algebra $\mathfrak{h}$ of $H$, we have

$$
\mathfrak{h} \cong \mathfrak{g}_{0} \oplus \mathfrak{g}_{1} \text {. }
$$

For $\mathbf{e} \in Q^{\prime}$, we decompose

$$
T_{\mathbf{e}} Q=T_{\mathbf{e}}^{V} \oplus T_{\mathbf{e}}^{H}=g_{-1} \oplus g_{0} \oplus g_{1} \oplus T_{\mathbf{e}}^{H} .
$$

We define

$$
\tilde{J}_{T}: T_{\mathbf{e}} Q \rightarrow T_{\mathbf{e}} Q
$$

by the following way:

$$
\begin{aligned}
& \text { (i) } \tilde{J}_{T}\left(\mathfrak{g}_{0}\right)=0, \\
& \text { (ii) } \tilde{J}_{T}\left(X_{2}\right)=X_{3}, \tilde{J}_{T}\left(X_{3}\right)=-X_{2}, \\
& \text { (iii) } \tilde{J}_{T}\left(Y_{2}\right)=-Y_{3}, \tilde{J}_{T}\left(Y_{3}\right)=Y_{2}, \\
& \text { (iv) }\left.\tilde{J}_{T}\right|_{T_{\mathrm{e}}^{H}}=\bar{J}_{T},
\end{aligned}
$$

where we write the fundamental vector fields $X_{i}^{*}, Y_{i}^{*}$ simply by $X_{i}, Y_{\imath}$. We have $\tilde{J}_{T}\left(W_{1}\right)=\tilde{J}_{T}\left(X_{2}+Y_{3}\right)=X_{3}+Y_{2}=W_{2}, \quad \tilde{J}_{T}\left(W_{2}\right)=\tilde{J}_{T}\left(X_{3}+Y_{2}\right)=-X_{2}-Y_{3}=-W_{1}$.

Lemma. $\quad \tilde{J}_{T}$ is $S O(2)$ equivariant, that is, for $a \in S O(2) \subset S O_{+}(3,1)$, 


$$
R_{a *} \tilde{J}_{T}=\tilde{J}_{T} R_{a *}
$$

Proof. As it follows easily that $R_{a *} \tilde{J}_{T}\left(X_{\imath}\right)=\tilde{J}_{T} R_{a *}\left(X_{\imath}\right), i=2,3$, we will prove $R_{a *} \tilde{J}_{T}\left(Y_{i}\right)=\tilde{J}_{T} R_{a *}\left(Y_{\imath}\right), i=2,3$. Take

$$
a=\left(\begin{array}{cccc}
1 & 0 & 0 & 0 \\
0 & 1 & 0 & 0 \\
0 & 0 & \cos \theta & -\sin \theta \\
0 & 0 & \sin \theta & \cos \theta
\end{array}\right) .
$$

Since

$$
\begin{aligned}
& R_{a *} Y_{2}=\operatorname{Ad}\left(a^{-1}\right) Y_{2}=\sin \theta Y_{3}+\cos \theta Y_{2}, \\
& R_{a *} Y_{3}=\operatorname{Ad}\left(a^{-1}\right) Y_{3}=\cos \theta Y_{3}-\sin \theta Y_{2},
\end{aligned}
$$

therefore

$$
\begin{aligned}
R_{a *} \tilde{J}_{T}\left(Y_{2}\right) & =-R_{a *} Y_{3}=-\cos \theta Y_{3}+\sin \theta Y_{2}, \\
\tilde{J}_{T} R_{a *}\left(Y_{2}\right) & =\tilde{J}_{T}\left(\sin \theta Y_{3}+\cos \theta Y_{2}\right)=\sin \theta \tilde{J}_{T}\left(Y_{3}\right)+\cos \theta \tilde{J}_{T}\left(Y_{2}\right) \\
& =\sin \theta Y_{2}-\cos \theta Y_{3} .
\end{aligned}
$$

Therefore we obtain that $R_{a *} \tilde{J}_{T}\left(Y_{2}\right)=\tilde{J}_{T} R_{a *}\left(Y_{2}\right)$. Similarly we obtain that $R_{a *} \tilde{J}_{T}\left(Y_{3}\right)=\tilde{J}_{T} R_{a *}\left(Y_{3}\right)$.

LEMma. We have

$$
\begin{gathered}
\Phi_{*} \tilde{J}_{T}=J_{T} \Phi_{*}, \\
\operatorname{Ker} \Phi_{*}=\mathfrak{h}=\mathfrak{g}_{0} \oplus \mathfrak{g}_{1} .
\end{gathered}
$$

Proof. According to the definitions of horizontal spaces and horizontal lifts, the horizontal space in $Q$ at a point on $Q^{\prime}$ is isomorphically mapped onto the horizontal space at a point on $P: \Phi_{*} T^{H}=T^{h}$. To be precise, $\Phi_{*}\left(e_{i}^{H}\right)=e_{i}^{h}, i=$ $1,2,3,4$, hold. Therefore the conclusion follows from the lemma above.

4. Since we can regard $\Phi: Q^{\prime} \rightarrow P$ as a quotient mapping by the right action of $\left\{a(\theta)=\exp \left(\theta Y_{1}\right)\right\} \cong S O(2)$, the following proposition is a consequence of the above lemma.

PROPOSITION. The following conditions are equivalent:

(1) $J_{T}$ is integrable on $P$. 
(2) For Neijenhuis tensor $N$, we have

$$
N(U, V)=[U, V]-[J U, J V]+J[J U, V]+J[U, J V]=0,
$$

where $U, V$ are vector fields on $P$.

(3) For $\tilde{J}=\tilde{J}_{T}$ on $Q$, we have

$$
\tilde{N}(\tilde{U}, \tilde{V})=[\tilde{U}, \tilde{V}]-[\tilde{J} \tilde{U}, \tilde{J} \tilde{V}]+\tilde{J}[\tilde{J} \tilde{U}, \tilde{V}]+\tilde{J}[\tilde{U}, \tilde{J} \tilde{V}] \subset \operatorname{span}\left\{X_{1}, Y_{1} ; V_{1}, V_{2}\right\},
$$

where $\tilde{U}, \tilde{V}$ are vector fields on $Q$ along $Q^{\prime}$ and $\Phi$-related with $U, V$ respectively, which is equivalent to that

$$
\tilde{N}(\tilde{U}, \tilde{V}) \equiv 0 \bmod \operatorname{span}\left\{X_{1}, Y_{1} ; V_{1}, V_{2}\right\},
$$

where $\tilde{U}, \tilde{V}$ belong to $\operatorname{span}\left\{W_{1}, W_{2}\right\} \oplus T^{H}$.

From now on, we write $\tilde{N}$ and $\tilde{J}$ simply by $N$ and $J$.

\section{§3. Proof of Theorem}

In the following we investigate the curvature condition with respect to $g$ of $M$ such that $N \equiv 0 \bmod \operatorname{span}\left\{X_{1}, Y_{1} ; V_{1}, V_{2}\right\}$ by dividing into several parts.

1. We have the following relations:

$$
\begin{aligned}
& {\left[X_{1}, X_{2}\right]=-Y_{3}, \quad\left[X_{1}, X_{3}\right]=-Y_{2}, \quad\left[X_{2}, X_{3}\right]=-Y_{1},} \\
& {\left[X_{1}, Y_{1}\right]=0, \quad\left[X_{1}, Y_{2}\right]=-X_{3}, \quad\left[X_{1}, Y_{3}\right]=-X_{2},} \\
& {\left[X_{2}, Y_{1}\right]=-X_{3}, \quad\left[X_{2}, Y_{2}\right]=0, \quad\left[X_{2}, Y_{3}\right]=X_{1},} \\
& {\left[X_{3}, Y_{1}\right]=X_{2}, \quad\left[X_{3}, Y_{2}\right]=X_{1}, \quad\left[X_{3}, Y_{3}\right]=0,} \\
& {\left[Y_{1}, Y_{2}\right]=-Y_{3}, \quad\left[Y_{1}, Y_{3}\right]=Y_{2}, \quad\left[Y_{2}, Y_{3}\right]=-Y_{1} .}
\end{aligned}
$$

The proof of the following is straightforward.

LEMMA. We have

$$
N\left(W_{1}, W_{2}\right)=0
$$

2. Let $x$ be a point in $M$ and let $\mathbf{e}=\left(e_{1}=T ; e_{2}, e_{3}, e_{4}\right)$ be a $T$-Lorentz orthonormal basis on $T_{x} M$. Choose an open subset $U \subset M$ such that the point $x$ belongs to $U$ and the linear frame bundle $Q^{\prime}$ is a trivial bundle over $U$. Let

$$
\mathbf{s}=\left(s_{1}=T ; s_{2}, s_{3}, s_{4}\right): U \rightarrow Q^{\prime}
$$


be a set of local sections such that $s_{1}=T ; s_{2}, s_{3}, s_{4}$ are Lorentz orthonormal frame fields on $U$ and $\mathbf{s}(x)=\mathbf{e}$, i.e., $s_{i}(x)=e_{i}, i=1,2,3,4$, hold. Put

$$
Z_{i}=s_{i}^{H}=s_{i}-\sum_{j, k} \omega_{j k}\left(s_{\imath}\right) E_{\jmath k}, \quad i=1,2,3,4 .
$$

Here we write $s_{*} s_{i}$ and $E_{j k}^{*}$ simply by $s_{i}$ and $E_{j k}$. The $S O_{+}(3,1)$-connection form $\omega=\left(\omega_{j k}\right)$ is defined by $\nabla s_{\jmath}=\sum_{k} \omega_{j k} \otimes s_{k}$, where $\nabla$ is the Levi-Civita connection. Since $\omega$ is a $\mathrm{o}(3,1)$-valued 1 -form, we can write

$$
\omega=\left(\begin{array}{cccc}
0 & \omega_{12} & \omega_{13} & \omega_{14} \\
\omega_{12} & 0 & \omega_{23} & \omega_{24} \\
\omega_{13} & -\omega_{23} & 0 & \omega_{34} \\
\omega_{14} & -\omega_{24} & -\omega_{34} & 0
\end{array}\right)
$$

for

$$
\omega_{12}=\omega_{21}, \omega_{13}=\omega_{31}, \omega_{14}=\omega_{41} ; \omega_{23}=-\omega_{32}, \omega_{24}=-\omega_{42}, \omega_{34}=-\omega_{43} .
$$

We have

$$
T^{H}=\operatorname{span}\left\{Z_{1} ; Z_{2}, Z_{3}, Z_{4}\right\}
$$

Since we have

$$
Z_{i}=e_{i}^{H}, i=1,2,3,4,
$$

at $\mathbf{e}=\left(e_{1}=T, e_{2}, e_{3}, e_{4}\right)$ in $Q^{\prime}$,

$$
J\left(Z_{1}\right)=Z_{2}, \quad J\left(Z_{2}\right)=-J\left(Z_{1}\right) ; J\left(Z_{3}\right)=Z_{4}, \quad J\left(Z_{4}\right)=-Z_{3}
$$

hold on $T_{\mathbf{e}}^{H}$. From the 3 -rd step to the 6 -th step, we will discuss the problem at $\mathbf{e} \in Q^{\prime}$.

3. We will show the following lemma:

Lemma. We have

$$
N\left(Z_{i}, W_{1}\right) \equiv 0 \text { and } N\left(Z_{i}, W_{2}\right) \equiv 0 \bmod \left\{X_{1}, Y_{1} ; V_{1}, V_{2}\right\}, i=1,2,3,4 .
$$

Proof. We will only prove the former equation since the latter is proved similarly. 


$$
\begin{aligned}
N\left(Z_{1}, W_{1}\right)=[ & \left.Z_{1}, W_{1}\right]-\left[Z_{2}, W_{2}\right]+J\left[Z_{2}, W_{1}\right]+J\left[Z_{1}, W_{2}\right] \\
= & \left.s_{1}-\sum \omega_{i j}\left(s_{1}\right) E_{\imath \jmath}, W_{1}\right]-\left[s_{2}-\sum \omega_{\imath j}\left(s_{2}\right) E_{i j}, W_{2}\right] \\
& +J\left[s_{2}-\sum \omega_{i j}\left(s_{2}\right) E_{\imath \jmath}, W_{1}\right]+J\left[s_{1}-\sum \omega_{i j}\left(s_{1}\right) E_{i j}, W_{2}\right] \\
= & -\sum \omega_{\imath j}\left(s_{1}\right)\left[E_{i j}, W_{1}\right]+\sum \omega_{i j}\left(s_{2}\right)\left[E_{i j}, W_{2}\right] \\
& -J\left(\sum \omega_{\imath j}\left(s_{2}\right)\left[E_{\imath j}, W_{1}\right]\right)-J\left(\sum \omega_{\imath j}\left(s_{1}\right)\left[E_{i j}, W_{2}\right]\right) \\
=- & \left(\omega_{12}\left(s_{1}\right)\left[X_{1}, W_{1}\right]+\omega_{13}\left(s_{1}\right)\left[X_{2}, W_{1}\right]+\omega_{14}\left(s_{1}\right)\left[X_{3}, W_{1}\right]\right. \\
& \left.+\omega_{23}\left(s_{1}\right)\left[-Y_{3}, W_{1}\right]+\omega_{24}\left(s_{1}\right)\left[-Y_{2}, W_{1}\right]+\omega_{34}\left(s_{1}\right)\left[-Y_{1}, W_{1}\right]\right) \\
+ & \omega_{12}\left(s_{2}\right)\left[X_{1}, W_{2}\right]+\omega_{13}\left(s_{2}\right)\left[X_{2}, W_{2}\right]+\omega_{14}\left(s_{2}\right)\left[X_{3}, W_{2}\right] \\
& \left.+\omega_{23}\left(s_{2}\right)\left[-Y_{3}, W_{2}\right]+\omega_{24}\left(s_{2}\right)\left[-Y_{2}, W_{2}\right]+\omega_{34}\left(s_{2}\right)\left[-Y_{1}, W_{2}\right]\right) \\
- & J\left(\omega_{12}\left(s_{2}\right)\left[X_{1}, W_{1}\right]+\omega_{13}\left(s_{2}\right)\left[X_{2}, W_{1}\right]+\omega_{14}\left(s_{2}\right)\left[X_{3}, W_{1}\right]\right. \\
& \left.+\omega_{23}\left(s_{2}\right)\left[-Y_{3}, W_{1}\right]+\omega_{24}\left(s_{2}\right)\left[-Y_{2}, W_{1}\right]+\omega_{34}\left(s_{2}\right)\left[-Y_{1}, W_{1}\right]\right) \\
- & J\left(\omega_{12}\left(s_{1}\right)\left[X_{1}, W_{2}\right]+\omega_{13}\left(s_{1}\right)\left[X_{2}, W_{2}\right]+\omega_{14}\left(s_{1}\right)\left[X_{3}, W_{2}\right]\right. \\
& \left.+\omega_{23}\left(s_{1}\right)\left[-Y_{3}, W_{2}\right]+\omega_{24}\left(s_{1}\right)\left[-Y_{2}, W_{2}\right]+\omega_{34}\left(s_{1}\right)\left[-Y_{1}, W_{2}\right]\right) \\
= & \left(\omega_{12}\right) W_{1}+\omega_{34}\left(s_{1}\right) W_{2}-\omega_{12}\left(s_{2}\right) W_{2}+\omega_{34}\left(s_{2}\right) W_{1} \\
+ & J\left(\omega_{12}\left(s_{2}\right) W_{1}\right)+J\left(\omega_{34}\left(s_{2}\right) W_{2}\right)+J\left(\omega_{12}\left(s_{1}\right) W_{2}\right)-J\left(\omega_{34}\left(s_{1}\right) W_{1}\right) \\
& +\{\ldots\} \quad\left(\{\ldots\} \text { is the terms with respect to } X_{1}, Y_{1} ; V_{1}, V_{2}\right) \\
\equiv 0 & \bmod \left\{X_{1}, Y_{1} ; V_{1}, V_{2}\right\} . \\
& \{
\end{aligned}
$$

Similarly we obtain that

$$
N\left(Z_{i}, W_{1}\right) \equiv 0 \bmod \left\{X_{1}, Y_{1} ; V_{1}, V_{2}\right\}, i=2,3,4 .
$$

4. The following equations are easy to see.

Lemma. We have

$$
\begin{aligned}
& N\left(Z_{1}, Z_{2}\right)=0, \quad N\left(Z_{3}, Z_{4}\right)=0 \\
& N\left(Z_{1}, Z_{3}\right)=-N\left(Z_{2}, Z_{4}\right), \quad N\left(Z_{1}, Z_{4}\right)=N\left(Z_{2}, Z_{3}\right) .
\end{aligned}
$$

5. From the above lemmas it remains to consider $N\left(Z_{1}, Z_{3}\right), N\left(Z_{1}, Z_{4}\right)$. For the above Lorentz orthonormal frame fields $s_{1}=T ; s_{2}, s_{3}, s_{4}$ on $U$, moreover let them satisfy $\left(\nabla s_{i}\right)_{x}=0$. They form a geodesic coordinate system at $x$. Therefore $\omega_{i j}\left(s_{k}\right)(x)=0$, and $\left.\left[s_{l}, s_{k}\right]\right|_{x}=\nabla_{s_{l}} s_{k}-\left.\nabla_{s_{k}} s_{l}\right|_{x}=0$.

For the curvature tensor $R$,

$$
R\left(s_{l}, s_{k}\right) s_{i}=\nabla_{s_{l}} \nabla_{s_{k}} s_{i}-\nabla_{s_{k}} \nabla_{s_{l}} s_{i}-\nabla_{\left[s_{l}, s_{k}\right]} s_{i} .
$$


Since $\nabla s_{i}=\sum \omega_{i j} s_{j}, \nabla_{s_{k}} s_{i}=\sum \omega_{i j}\left(s_{k}\right) s_{j}$, we have

$$
\nabla_{s_{l}} \nabla_{s_{k}} s_{i}=\sum s_{l} \omega_{i j}\left(s_{k}\right) s_{j}+\sum \omega_{i j}\left(s_{k}\right) \omega_{j m}\left(s_{l}\right) s_{m} .
$$

Similarly we have

$$
\nabla_{s_{k}} \nabla_{s_{l}} s_{i}=\sum s_{k} \omega_{i j}\left(s_{l}\right) s_{j}+\sum \omega_{i j}\left(s_{l}\right) \omega_{j m}\left(s_{k}\right) s_{m} .
$$

Hence

$$
\begin{aligned}
R_{l k i j}(x) & =g\left(R\left(s_{l}, s_{k}\right) s_{i}, s_{j}\right)(x) \\
& =g\left(\sum_{m}\left(s_{l} \omega_{l m}\left(s_{k}\right)-s_{k} \omega_{\imath m}\left(s_{l}\right)\right) s_{m}, s_{\jmath}\right)(x) \\
& =\left\{\begin{array}{l}
-s_{l} \omega_{i 1}\left(s_{k}\right)+s_{k} \omega_{i 1}\left(s_{l}\right), \quad \text { if } j=1, \\
s_{l} \omega_{i j}\left(s_{k}\right)-s_{k} \omega_{i j}\left(s_{l}\right), \quad \text { if } j=2,3,4 .
\end{array}\right.
\end{aligned}
$$

Consequently

$$
\begin{aligned}
{\left.\left[Z_{i}, Z_{j}\right]\right|_{\mathbf{e}}=} & {\left.\left[s_{i}-\sum \omega_{l k}\left(s_{i}\right) E_{l k}, s_{j}-\sum \omega_{m n}\left(s_{j}\right) E_{m n}\right]\right|_{\mathbf{e}} } \\
= & -\sum s_{\imath} \omega_{m n}\left(s_{j}\right) E_{m n}+\sum s_{j} \omega_{l k}\left(s_{i}\right) E_{l k} \\
= & \left(s_{i} \omega_{23}\left(s_{j}\right)-s_{j} \omega_{23}\left(s_{i}\right)\right) Y_{3}+\left(s_{i} \omega_{24}\left(s_{j}\right)-s_{j} \omega_{24}\left(s_{i}\right)\right) Y_{2} \\
& +\left(-s_{i} \omega_{13}\left(s_{j}\right)+s_{j} \omega_{13}\left(s_{i}\right)\right) X_{2}+\left(-s_{i} \omega_{14}\left(s_{j}\right)+s_{j} \omega_{14}\left(s_{i}\right)\right) X_{3}+\{\ldots\} \\
= & R_{i j 23} Y_{3}+R_{i j 24} Y_{2}+R_{i j 31} X_{2}+R_{i j 41} X_{3}+\{\ldots\} \\
= & \frac{1}{2}\left(R_{i j 23}+R_{i j 31}\right) W_{1}+\frac{1}{2}\left(R_{i j 24}+R_{i j 41}\right) W_{2} \\
& +\frac{1}{2}\left(-R_{i j 23}+R_{i j 31}\right) V_{1}+\frac{1}{2}\left(-R_{i j 24}+R_{i j 41}\right) V_{2}+\{\ldots\} \text { (e is omitted). }
\end{aligned}
$$

6. Finally we examine $N\left(Z_{1}, Z_{3}\right), N\left(Z_{1}, Z_{4}\right)$.

Lemma. The following conditions are equivalent:

$$
\begin{gathered}
N\left(Z_{1}, Z_{3}\right) \equiv 0 \bmod \left\{X_{1}, Y_{1} ; V_{1}, V_{2}\right\} \\
N\left(Z_{1}, Z_{4}\right) \equiv 0 \bmod \left\{X_{1}, Y_{1} ; V_{1}, V_{2}\right\} \\
\left\{\begin{array}{l}
R_{1323}+R_{1331}+R_{1324}-2 R_{2324}+R_{1423}+R_{1414}+R_{1442}=0 \\
R_{2323}+R_{2331}+R_{2314}-2 R_{1314}+R_{2413}+R_{2414}+R_{2442}=0 .
\end{array}\right.
\end{gathered}
$$

Proof. We have

$$
N\left(Z_{1}, Z_{3}\right)=\left[Z_{1}, Z_{3}\right]-\left[Z_{2}, Z_{4}\right]+J\left[Z_{2}, Z_{3}\right]+J\left[Z_{1}, Z_{4}\right]
$$




$$
\begin{aligned}
= & \frac{1}{2}\left(R_{1323}+R_{1331}\right) W_{1}+\frac{1}{2}\left(R_{1324}+R_{1341}\right) W_{2} \\
& -\frac{1}{2}\left(R_{2423}+R_{2431}\right) W_{1}-\frac{1}{2}\left(R_{2424}+R_{2441}\right) W_{2} \\
& +J\left(\frac{1}{2}\left(R_{2323}+R_{2331}\right) W_{1}+\frac{1}{2}\left(R_{2324}+R_{2341}\right) W_{2}\right) \\
& +J\left(\frac{1}{2}\left(R_{1423}+R_{1431}\right) W_{1}+\frac{1}{2}\left(R_{1424}+R_{1441}\right) W_{2}\right)+\{\ldots\} \\
= & \frac{1}{2}\left(R_{1323}+R_{1331}+R_{1324}-2 R_{2324}+R_{1423}+R_{1414}+R_{1442}\right) W_{1} \\
& +\frac{1}{2}\left(R_{2323}+R_{2331}+R_{2314}-2 R_{1314}+R_{2413}+R_{2414}+R_{2442}\right) W_{2}+\{\ldots\} .
\end{aligned}
$$

Therefore the necessary and sufficient condition for

$$
N\left(Z_{1}, Z_{3}\right) \equiv 0 \bmod \left\{X_{1}, Y_{1} ; V_{1}, V_{2}\right\}
$$

is that

$$
\left\{\begin{array}{l}
R_{1323}+R_{1331}+R_{1324}-2 R_{2324}+R_{1423}+R_{1414}+R_{1442}=0 \\
R_{2323}+R_{2331}+R_{2314}-2 R_{1314}+R_{2413}+R_{2414}+R_{2442}=0
\end{array}\right.
$$

Next we have

$$
\begin{aligned}
N\left(Z_{1}, Z_{4}\right)= & \frac{1}{2}\left(R_{2314}+R_{2323}+R_{2331}-2 R_{1314}+R_{2442}+R_{2414}+R_{2413}\right) W_{1} \\
& +\frac{1}{2}\left(R_{1424}+R_{1441}+R_{1432}+2 R_{2324}+R_{1342}+R_{1332}+R_{1313}\right) W_{2}+\{\ldots\} .
\end{aligned}
$$

Therefore the necessary and sufficient condition for

$$
N\left(Z_{1}, Z_{4}\right) \equiv 0 \bmod \left\{X_{1}, Y_{1} ; V_{1}, V_{2}\right\},
$$

is that

$$
\left\{\begin{array}{l}
R_{2314}+R_{2323}+R_{2331}-2 R_{1314}+R_{2442}+R_{2414}+R_{2413}=0 \\
R_{1424}+R_{1441}+R_{1432}+2 R_{2324}+R_{1342}+R_{1332}+R_{1313}=0
\end{array}\right.
$$

These are the same relations as for $N\left(Z_{1}, Z_{3}\right)$.

7. Up to now, for the fixed point $x \in M$, we have expressed the integrability of $J_{T}$ at a point $\mathbf{e}=\left(e_{1}=T ; e_{2}, e_{3}, e_{4}\right)$ with the distinguished local frame field $\mathbf{s}=\left(s_{1}=T ; s_{2}, s_{3}, s_{4}\right): U \rightarrow Q^{\prime}$ on the neighborhood $U$ of $x$ in $M$. We 
discuss the integrability of $J_{T}$ at all points along the fibre $\varpi^{-1}(x) \cong S O(3)$ by rotating e by $S O(3)$. First we even-permute the index $2,3,4$ in the two relations at (3) in lemma in 6-th step. We discuss the integrability of $J_{T}$ at $\mathbf{e}^{\prime}=\left(e_{1}=T\right.$; $\left.e_{4}, e_{2}, e_{3}\right)$ and $\mathbf{e}^{\prime \prime}=\left(e_{1}=T ; e_{3}, e_{4}, e_{2}\right)$ in $Q^{\prime}$. For the first relation,

$$
\begin{aligned}
& \left(\begin{array}{l}
234 \\
234
\end{array}\right): R_{1323}+R_{1331}+R_{1324}-2 R_{2324}+R_{1423}+R_{1414}+R_{1442}=0 \\
& \left(\begin{array}{l}
234 \\
423
\end{array}\right): R_{1242}+R_{1221}+R_{1243}-2 R_{4243}+R_{1342}+R_{1313}+R_{1334}=0 \\
& \left(\begin{array}{l}
234 \\
342
\end{array}\right): R_{1434}+R_{1441}+R_{1432}-2 R_{3432}+R_{1234}+R_{1212}+R_{1223}=0
\end{aligned}
$$

For the second relation,

$$
\begin{aligned}
& \left(\begin{array}{l}
234 \\
234
\end{array}\right): R_{2323}+R_{2331}+R_{2314}-2 R_{1314}+R_{2413}+R_{2414}+R_{2442}=0 \\
& \left(\begin{array}{c}
234 \\
423
\end{array}\right): R_{4242}+R_{4221}+R_{4213}-2 R_{1213}+R_{4312}+R_{4313}+R_{4334}=0 \\
& \left(\begin{array}{c}
234 \\
342
\end{array}\right): R_{3434}+R_{3441}+R_{3412}-2 R_{1412}+R_{3214}+R_{3212}+R_{3223}=0
\end{aligned}
$$

Thus we have obtained six relations of the curvature of the Lorentzian manifold which is equivalent to the relations of Theorem in $\S 1$. Next we rotate $\mathbf{e}$ by an arbitrary element $a \in S O(3)$. The two relations are changed by the action of $a \in S O$ (3). Using the skew-symmetry relations of the curvature tensor and representing $a \in S O$ (3) by Euler's angles, we can show that these two relations are linear combinations of the above six relations. This completes the proof of Theorem.

We note that the space of algebraic curvature tensors which satisfy the above six relations is $S O(3)$-invariant. See Remark A in $\S 5$.

\section{§4. Global examples}

1. Using Corollary in $\S 1$, we will give global examples of $M$ with a future-pointing timelike vector field $T$ such that $J_{T}$ is integrable on $P$.

If $M$ is of constant curvature and $T$ is an arbitrary future-pointing timelike vector field on $M$, then we have 


$$
Q=\left(\begin{array}{cc}
-\alpha I_{3} & O \\
O & \alpha I_{3}
\end{array}\right) \text { and } R=\alpha I_{6}=\left(\begin{array}{cc}
\alpha I_{3} & O \\
O & \alpha I_{3}
\end{array}\right)
$$

This form satisfies (1) of Corollary in $\S 1$ and $J_{T}$ is integrable on $P$.

If $M$ is the (Lorentz) product of 3 -dimensional Riemannian manifold $N$ with constant curvature and $\mathbf{R}_{1}^{1}$, then $M$ is conformally flat. Take $T$ to be the $\mathbf{R}_{1}^{1}$ direction. We have

$$
Q=\left(\begin{array}{cc}
O & O \\
O & \alpha I_{3}
\end{array}\right) \text { and } R=\left(\begin{array}{cc}
O & O \\
O & \alpha I_{3}
\end{array}\right)
$$

This form satisfies (2) of Corollary in $\S 1$ and $J_{T}$ is integrable on $P$.

Including two examples above, there are Robertson-Walker space-times with

$$
Q=\left(\begin{array}{cc}
\beta I_{3} & O \\
O & \alpha I_{3}
\end{array}\right)
$$

They are conformally flat. See [O'N]. Of course, there are many examples of conformally flat spaces which are not Robertson-Walker space-times.

Robertson-Walker space-time $M=M(k, f)$ is defined by (Lorentz) warped product $I \times{ }_{f} N$, where $I=(a, b) \subset \mathbf{R}_{1}^{1}(-\infty \leq a<b \leq+\infty)$ and $N$ is 3 -dimensional connected Riemannian manifold with constant curvature $k$. We write the Lorentzian metric $g$ as

$$
d s^{2}=-d t^{2}+f(t)^{2} d \sigma^{2},
$$

where $-d t^{2}$ is a line element on base manifold $I, d \sigma^{2}$ is a line element on fibre manifold $N$, and $f(t)>0$ is a smooth function on $I$ called a warping function or scaling function. Robertson-Walker space-time is a globally hyperbolic space, and spacelike slice $S(t)(t \in I)$ is a Cauchy hypersurface.

Especially if $f=1, M$ is the (Lorentz) product of 3-dimensional Riemannian manifold $N$ with constant curvature and $I$. This is Einstein's static universe. There are other special examples of Robertson-Walker space-times which are Lorentzian manifolds with constant curvature. We have $S_{1}^{4}=\mathbf{R}_{1}^{1} \times{ }_{f} S^{3}$, with $f=\cosh t$, called the de Sitter space-time. Using the polar coordinate system on $S^{3}$

$$
d s^{2}=-d t^{2}+\cosh ^{2} t\left(d r^{2}+\sin ^{2} r\left(d \theta^{2}+\sin ^{2} \theta d \phi^{2}\right)\right) .
$$

We have the flat space time $\mathbf{R}_{1}^{4}=\mathbf{R}_{1}^{1} \times \mathbf{R}^{3}$. We have $(0, \pi) \times{ }_{f} H^{3}$ for some warping function $f$ which is isometric to the half of the anti-de Sitter space $H_{1}^{4}$ diffeomorphic to $\mathbf{R}^{3} \times S^{1}$. Remark that the universal covering space $\tilde{H}_{1}^{4}$ of $H_{1}^{4}$ is 
given by $\tilde{H}_{1}^{4}=H^{3} \times{ }_{g} \mathbf{R}_{1}^{1}$, and $g=\cosh r$. Using the polar coordinate system on $H^{3}$,

$$
d s^{2}=-\cosh ^{2} r d t^{2}+d r^{2}+\sinh ^{2} r\left(d \theta^{2}+\sin ^{2} \theta d \phi^{2}\right) .
$$

On a Robertson-Walker space-time $M$, let us choose

$$
T=\frac{\partial}{\partial t}
$$

It is a unit vector field Lorentz orthogonal to each spacelike slice $S(t)$ and future-pointing with respect to the canonical timelike orientation. Let $X, Y, Z$ be vector fields tangent to $S(t)$, then we have

$$
\begin{aligned}
& R(X, Y) Z=-\left(\left(\frac{f^{\prime}}{f}\right)^{2}+\frac{k}{f^{2}}\right)(g(X, Z) Y-g(Y, Z) X) \\
& R(X, Y) T=-\frac{f^{\prime \prime}}{f} X, \quad R(X, Y) T=0, \quad R(X, Z) Y=-\frac{f^{\prime \prime}}{f} g(X, Y) T .
\end{aligned}
$$

The sectional curvature $K_{T}$ including $T$ satisfies $K_{T}=\frac{f^{\prime \prime}}{f}$. If $t$ is fixed, its values are constant. The sectional curvature $K_{\sigma}$ tangent to spacelike slice satisfies $K_{\sigma}=$ $\frac{f^{\prime 2}+k}{f^{2}}$. If $t$ is fixed, its values are constant. The Ricci curvature (tensor) has the following values:

$$
\begin{aligned}
& \operatorname{Ric}(T, T)=-\frac{3 f^{\prime \prime}}{f}, \quad \operatorname{Ric}(T, X)=0, \\
& \operatorname{Ric}(X, Y)=\left(2\left(\frac{f^{\prime}}{f}\right)^{2}+\frac{2 k}{f^{2}}+\frac{f^{\prime \prime}}{f}\right) g(X, Y) .
\end{aligned}
$$

The scalar curvature satisfies $S=\frac{1}{6}\left(\left(\frac{f^{\prime}}{f}\right)^{2}+\frac{k}{f^{2}}+\frac{f^{\prime \prime}}{f}\right)$. It follows that $M$ is conformally flat. If $s_{1}=T$ and $s_{2}=X, s_{3}=Y, s_{4}=Z$ are orthonormal vector tangent to $S(t)$, they are $T$-Lorentz orthonormal basis at a point in $M$. Then we have the following form $Q$ :

$$
Q=\left(\begin{array}{cc}
-\frac{f^{\prime \prime}}{f} I_{3} & 0 \\
O & \frac{f^{\prime 2}+k}{f^{2}} I_{3}
\end{array}\right) .
$$

This form satisfies (2) of Corollary in $\S 1$. 
Proposition. Suppose that $M$ is Robertson-Walker space-time. If we choose $T=$ $\frac{\partial}{\partial t}$ on $M$, then $J_{T}$ is integrable on $P$.

2. We remark that, if there is an example of $M$ with a form

$$
Q=\left(\begin{array}{cccccc}
-x & 0 & 0 & x & 0 & 0 \\
0 & -y & 0 & 0 & y & 0 \\
0 & 0 & x+y & 0 & 0 & -x-y \\
x & 0 & 0 & -x & 0 & 0 \\
0 & y & 0 & 0 & -y & 0 \\
0 & 0 & -x-y & 0 & 0 & x+y
\end{array}\right)
$$

then $M$ is not conformally flat, but $P$ is an integrable twistor space.

We remark that, if we choose the canonical $T$ on $M$ with Taub-NUT metric or Eguchi-Hanson metric, then $J_{T}$ is not integrable on $P$. Then, note that $M$ is Einstein.

\section{§5. Final remarks}

\section{Remark A:}

1. In 3 of $\S 1$, we considered the irreducible $S O_{+}(3,1)$-decomposition of algebraic curvature tensor space $\mathscr{R}$ on $\Lambda^{2}=\Lambda^{2} \mathbf{R}_{1}^{4}$. The vector space $\mathscr{R}$ is 20 -dimensional and consists of the three invariant subspaces. We obtained the corollary by the irreducible decomposition of the curvature tensor $R$ of $(M, g)$. Choose a future-pointing timelike unit vector field $T$ on $M$. Since

$$
S O_{+}(3,1) \supset S O(1) \times S O(3) \cong S O(3),
$$

we can regard $\mathscr{R}$ as an $S O(3)$-module. In this section, we subdecompose $\mathscr{R}$ into irreducible $S O(3)$-invariant subspaces and we will restate the conclusion of Theorem as the vanishing of certain components of the curvature tensor $\mathscr{R}$.

We have the action of $S O_{+}(3,1)$ on $\Lambda^{2}$ defined by

$$
\rho(a)(u \wedge v)=a u \wedge a v, \quad a \in S O_{+}(3,1) .
$$

Choose a $T$-Lorentz orthonormal basis $\mathbf{e}=\left(e_{1}=T ; e_{2}, e_{3}, e_{4}\right) \in T_{x} M$. Then we have the orthonormal basis $\tilde{\mathbf{e}}$ of $\Lambda^{2}$. With respect to this basis, the action $\rho$ defines an exterior product representation

$$
\rho: S O_{+}(3,1) \rightarrow G L\left(\Lambda^{2}\right)
$$


We can restrict the representation to obtain

$$
\rho: S O(3) \rightarrow G L\left(\Lambda^{2}\right) .
$$

Let

$$
a=\left(\begin{array}{cccc}
1 & 0 & 0 & 0 \\
0 & & & \\
0 & & & a^{\prime} \\
0 & & &
\end{array}\right)
$$

be an element in $S O(1) \times S O(3) \subset S O_{+}(3,1)$, where $a^{\prime} \in S O(3)$. Since $a e_{1}=$ $e_{1}, T=e_{1}$, we have

$$
\rho(a)=\left(\begin{array}{cc}
{ }^{t} a^{\prime} & O \\
O & { }^{t} a^{\prime}
\end{array}\right), \quad \rho(a)^{-1}=\left(\begin{array}{cc}
a^{\prime} & O \\
O & a^{\prime}
\end{array}\right) .
$$

We regard $\mathscr{R}$ as a subset of $\operatorname{Mat}\left(\Lambda^{2}\right) \cong \operatorname{Mat}(6)$, all $6 \times 6$ matrices. We have

$$
\begin{aligned}
\rho(a)^{-1} R \rho(a) & =\left(\begin{array}{cc}
a^{\prime} & O \\
O & a^{\prime}
\end{array}\right)\left(\begin{array}{cc}
-A & -B \\
{ }^{t} B & C
\end{array}\right)\left(\begin{array}{cc}
{ }^{t} a^{\prime} & O \\
O & { }^{t} a^{\prime}
\end{array}\right) \\
& =\left(\begin{array}{cc}
-a^{\prime} A^{t} a^{\prime} & -a^{\prime} B^{t} a^{\prime} \\
a^{\prime} B^{t} a^{\prime} & a^{\prime} C^{t} a^{\prime}
\end{array}\right) .
\end{aligned}
$$

The transformation defined by $\mathscr{R} \ni R \mapsto \rho(a)^{-1} R \rho(a)$ gives $\mathscr{R}$ the structure of an $S O(3)$-module. The $3 \times 3$ matrices $A, B, C$ are transformed by the conjugate transformations of $a^{\prime} \in S O(3)$.

Then, we can take another point of view. We regard $\mathscr{R}$ as a codimension one subspace of $S^{2}\left(\Lambda^{2}\right)$, the space of all symmetric 2-tensors on $\Lambda^{2}$. Then, in consideration of $\Lambda^{2}=\Lambda_{t}^{2} \oplus \Lambda_{s}^{2}$, we have the $S O(3)$-decomposition

$$
S^{2}\left(\Lambda^{2}\right)=S^{2}\left(\Lambda_{t}^{2}\right) \oplus\left(\Lambda_{t}^{2} \otimes \Lambda_{s}^{2}\right) \oplus S^{2}\left(\Lambda_{s}^{2}\right) .
$$

Therefore $R$ has the components decomposed into the above three $S O(3)$ invariant subspaces.

2. We recall the real representation theory of $S O(3)$. The real irreducible representations of $S O(3)$ are of odd degrees as well as that of the complex irreducible representations of $S O(3)$. In 1-, 3-, 5-dimensions, the representations are realized by conjugate transformations on the vector space Mat(3).

Lemma. The $S O(3)$-module Mat(3) is decomposed into the following three irre- 
ducible subspaces:

$$
\operatorname{Mat}(3)=\mathfrak{D} \oplus \mathfrak{S} \oplus \mathfrak{A},
$$

where $\mathfrak{D}$ is the set of all $3 \times 3$ scalar matrices and $\operatorname{dim} \mathfrak{D}=1$,

$\mathfrak{S}$ is the set of all $3 \times 3$ trace-free symmetric matrices and $\operatorname{dim} \mathfrak{D}=5$,

$\mathfrak{A}$ is the set of all $3 \times 3$ skew-symmetric matrices and $\operatorname{dim} \mathfrak{D}=3$.

Proof. First Mat(3) is decomposed into the symmetric part and the skewsymmetric part. In the set of all symmetric matrices Sym(3) which is 6 dimensional, for $A \in \operatorname{Sym}(3)$, we have

$$
\begin{aligned}
& A=\left(\begin{array}{lll}
a & d & e \\
d & b & f \\
e & f & c
\end{array}\right) \\
& =\left(\begin{array}{ccc}
\frac{a+b+c}{3} & 0 & 0 \\
0 & \frac{a+b+c}{3} & 0 \\
0 & 0 & \frac{a+b+c}{3}
\end{array}\right)+ \\
& \left(\begin{array}{ccc}
a-\frac{a+b+c}{3} & d & e \\
d & b-\frac{a+b+c}{3} & f \\
e & f & c-\frac{a+b+c}{3}
\end{array}\right)
\end{aligned}
$$

The first term is a scalar matrix and the second term a trace-free matrix. It follows easily that $\mathfrak{S}, \mathfrak{A}$ are irreducible by conjugate transformations of $S O(3)$.

Note that the representation of $S O(3)$ by conjugate transformations on Mat(3) is equivalent to the tensor product representation $\tau_{1} \otimes \tau_{1}$ on a 9-dimensional representation space. Here $\tau_{1}$ is the 3 -dimensional irreducible representation of $S O(3)$ induced by the 3 -dimensional irreducible representation $\rho_{2}$ with the highest weight 2 of $S U(2)$. By the way, according to the Clebsch-Gordan's formula, $\tau_{1} \otimes \tau_{1}$ is decomposed into the direct sum of the irreducible representations of $S O(3)$ :

$$
\tau_{1} \otimes \tau_{1}=\tau_{2} \oplus \tau_{1} \oplus \tau_{0}
$$


where $\operatorname{deg} \tau_{2}=5, \operatorname{deg} \tau_{1}=3$, deg $\tau_{0}=1$. This gives the irreducible decomposition of lemma.

3. As mentioned in $\S 1, R \in \mathscr{R}$ has the components representation decomposed into the three irreducible $\mathrm{SO}_{+}(3,1)$-invariant subspaces:

$$
R=\left(\begin{array}{cc}
-A & -B \\
{ }^{t} B & C
\end{array}\right)=W+L+U
$$

On the other hand, as mentioned above, $R$ can be regarded as $R=A+B+C$ by the components of not irreducible $S O(3)$-invariant subspaces. Put

$$
\begin{aligned}
\mathscr{A} & :=\left\{-A: \Lambda_{t}^{2} \rightarrow \Lambda_{t}^{2}, \text { self-adjoint linear transformation }\right\} \\
& \cong \operatorname{Sym}(3)=\mathscr{D} \oplus \mathbb{S}=: \mathscr{A}_{D} \oplus \mathscr{A}_{S}, \\
\mathscr{B} & :=\left\{-B: \Lambda_{s}^{2} \rightarrow \Lambda_{t}^{2}, \text { linear transformation, tr }=0\right\} \\
& \cong \mathfrak{S} \oplus \mathfrak{A}=: \mathscr{B}_{S} \oplus \mathscr{B}_{A}, \\
\mathscr{C} & :=\left\{C: \Lambda_{s}^{2} \rightarrow \Lambda_{s}^{2}, \text { self-adjoint linear transformation }\right\} \\
& \cong \operatorname{Sym}(3)=\mathscr{D} \oplus \mathfrak{S}=: \mathscr{C}_{D} \oplus \mathscr{C}_{S} .
\end{aligned}
$$

Then we have

$$
\begin{aligned}
\mathscr{R} & =\mathscr{A} \oplus \mathscr{B} \oplus \mathscr{C} \cdots S O(3) \text {-decomposition, } \\
& =\mathscr{A}_{D} \oplus \mathscr{A}_{S} \oplus \mathscr{B}_{S} \oplus \mathscr{B}_{A} \oplus \mathscr{C}_{D} \oplus \mathscr{C}_{S} \cdots \text { irreducible } S O(3) \text {-decomposition. }
\end{aligned}
$$

We can make new six irreducible $S O(3)$-invariant decomposition by transforming some basis. Then we can remark that $J_{T}$ is integrable on $P$ if and only if the two components of some 1 dimensional and 5 dimensional $S O(3)$-invariant subspaces of $R$ vanish.

\section{Remark B:}

1. We recall the construction of $[\mathrm{BB}-\mathrm{O}]$. Let $\mathscr{C}$ be the set of all complex structures on $\mathbf{R}^{2 n}$. A general linear group $G L(2 n, \mathbf{R})$ acts transitively on $\mathscr{C}$ by conjugation.

For a closed subgroup $G_{0}$ of $G L(2 n, \mathbf{R})$, let $C$ be a $G_{0}$-invariant submanifold of $\mathscr{C}$. We have $C \cong G_{0} / H_{0}$. We assume that $C$ has a $G_{0}$-invariant almost complex structure.

Next, let $M$ be a $2 n$-dimensional differentiable manifold, and let $P_{0}$ be a linear frame bundle over $M$ with structure group $G_{0}$ and a projection $\varpi: P_{0} \rightarrow M$. 
Moreover let $Z$ be a fibre bundle with fibre $C$ associated to the principal bundle $P_{0}$. Namely, we have $Z=P_{0} \times{ }_{G_{0}} C$ with a projection $\pi: Z \rightarrow M$.

For a $G_{0}$-comnection on $P_{0}$, we can consider the associated horizontal lift of $M$ to $Z$.

Proposition [BB-O]. There exists a natural almost complex structure $J_{0}$ on $Z$.

2. We generalize the above situation. We assume that $G(\subset G L(2 n, \mathbf{R}))$, including $G_{0}$ as a subgroup, acts on $C$ transitively and effectively and that the action of $G$ restricted to $G_{0}$ coincides with the above-mentioned conjugate action. Then we have $C \cong G / H \cong G_{0} / G_{0} \cap H \cong G_{0} / H_{0}$. Moreover we assume that a linear frame bundle $P$ with structure group $G$ is reduced to one with structure group $G_{0}$. Then we have $Z=P \times{ }_{G} C=P_{0} \times{ }_{G_{0}} C$. We assume that $C$ has a $G$-invariant almost complex structure $J_{1}$. For a $G$-connection on $P$, we can consider the associated horizontal lift of $M$ to $Z$. Under the above-mentioned assumptions, we get the following proposition.

Proposition. There exists an almost complex structure $J$ on $Z$ with respect to the G-connection.

Proof. The right action of $G_{0}$ on $P_{0} \times C$ is as follows. For $g_{0} \in G_{0}$,

$$
P_{0} \times C \ni\left(p_{0}, c\right) \mapsto\left(p_{0}, c\right) g_{0}=\left(p_{0} g_{0}, g_{0}^{-1} c\right) \in P_{0} \times C \text {. }
$$

Then $Z$ is the set of equivalence classes of $P_{0} \times C$ by the equivalence relation $~$ defined as follows:

$$
\left(p_{0}, c\right) \sim\left(q_{0}, d\right) \Leftrightarrow \text { there exists } g_{0} \in G_{0} \text { such that } q_{0}=p_{0} g_{0}, d=g_{0}^{-1} c .
$$

At $z=\left[\left(p_{0}, c\right)\right] \in Z$, the tangent space $T_{z} Z$ is the set of

$$
[(\xi, w)]=\left[\left(R_{g_{0} *} \xi, g_{0_{*}}^{-1} w\right)\right], g_{0} \in G_{0} \text {, for }(\xi, w) \in T_{\left(p_{0}, c\right)}\left(P_{0} \times C\right) \cong T_{p_{0}} P_{0} \times T_{c} C .
$$

With respect to the $G$-connection on $P_{0} \subset P, T P_{0} \subset T P$ is decomposed into the vertical bundle $V$ and the horizontal bundle $H$. Then the class

$$
\left[(0, v, 0) \in T_{\left(p_{0}, c\right)}\left(P_{0} \times C\right)=V_{p_{0}} \oplus H_{p_{0}} \oplus T_{c} C\right] \in T_{z} Z
$$

is equal to the class

$$
\left[\left(0, R_{g_{0} *} v, 0\right) \in T_{\left(p_{0} g_{0}, g_{0}^{-1} c\right)}\left(P_{0} \times C\right)=V_{p_{0} g_{0}} \oplus H_{p_{0} g_{0}} \oplus T_{g_{0}^{-1} c}\right] \in T_{z} Z .
$$

The class 


$$
\left[(0,0, w) \in V_{p_{0}} \oplus H_{p_{0}} \oplus T_{c} C\right] \in T_{z} Z
$$

is equal to the class

$$
\left[\left(0,0, g_{0 *}^{-1} w\right) \in V_{p_{0} g_{0}} \oplus H_{p_{0} g_{0}} \oplus T_{g_{0}^{-1} c} C\right] \in T_{z} Z .
$$

Therefore we have

$$
T_{z} Z \cong H_{p_{0}} \oplus T_{c} C
$$

We remark that, with respect to the $G$-connection, we have the decompositions

$$
T_{z} Z=\mathscr{H}_{z} \oplus \mathscr{V}_{z}, \quad \mathscr{H}_{z} \cong H_{p_{0}}, \quad \mathscr{V}_{z} \cong T_{c} C .
$$

Since $J_{1}$ is $G$-invariant, for $[(0,0, w)] \in \mathscr{V}_{z}$, we have the following commutative diagram: for $g \in G$,

$$
\begin{array}{ccc}
w \in T_{c} C & \stackrel{J_{1}}{\longrightarrow} & T_{c} C \ni J_{1} w \\
g^{-1} \downarrow & & \downarrow g^{-1} \\
g_{*}^{-1} w \in T_{g^{-1} c} C & \underset{J_{1}}{\longrightarrow} & T_{g^{-1} c} C \ni J_{1} g_{*}^{-1} w .
\end{array}
$$

Therefore we can define an almost complex structure $J_{1}$ on $\mathscr{V}_{z}$ and $\mathscr{V}=U_{z \in Z} \mathscr{V}_{z}$.

For $[(0, v, 0)] \in \mathscr{H}_{z}$, we will define an almost complex structure $J_{2}$. Remark that $c \in C$ is a complex structure on $\mathbf{R}^{2 n}$. Since we regard $p_{0} \in P_{0}$ as a linear isomorphism of $\mathbf{R}^{2 n}$ onto $T_{x} M\left(x=\varpi\left(p_{0}\right)\right)$, a mapping $p_{0} c p_{0}^{-1}$ defines a complex structure on $T_{x} M$. According to $T_{x} M \cong H_{p_{0}}$ with respect to the $G$-connection, we can define a complex structure $c^{h}$ on $H_{p_{0}}$. On the other hand, $g_{0}^{-1} c \in C\left(g_{0} \in G_{0}\right)$ is also a complex structure on $\mathbf{R}^{2 n}$. From $p_{0} g_{0} \in P_{0}$, a mapping $\left(p_{0} g_{0}\right)\left(g_{0}^{-1} c\right)$ $\left(p_{0} g_{0}\right)^{-1}$ defines a complex structure on $T_{x} M$. We define a complex structure $\left(g_{0}^{-1} c\right)^{h}$ on $H_{p_{0} g_{0}}$. We have the following commutative diagram: for $g_{0} \in G_{0}$,

$$
\begin{aligned}
& v \in H_{p_{0}} \stackrel{c^{h}}{\longrightarrow} \quad H_{p_{0}} \ni c^{h} v \\
& g_{0} \downarrow \quad \downarrow g_{0} \\
& R_{g_{0} *} \boldsymbol{v} \in H_{p_{0} g_{0}} \underset{\left(g_{0}^{-1} c\right)^{h}}{\longrightarrow} H_{p_{0} g_{0}} \ni\left(g_{0}^{-1} c\right)^{h}\left(R_{g_{0} *} v\right) \text {. }
\end{aligned}
$$

In fact, we will show that $\left(g_{0}^{-1} c\right)^{h}=R_{g_{0} *} c^{h} R_{g_{0}^{-1} *}$. From the assumption that the action of $G_{0}$ on $C$ is conjugation, we can conclude it. Namely, for $g_{0} \in G_{0}$, we have $g_{0}(c)=g_{0}^{-1} c g_{0}, g_{0}^{-1}(c)=g_{0} c g_{0}^{-1}$. Therefore we can define an almost complex structure $J_{2}$ on $\mathscr{H}_{z}$ and $\mathscr{H}$. Hence an almost complex structure $J=J_{1} \oplus J_{2}$ is defined on $Z$. 
3. This proposition generalizes the result stated before ([BB-O]). In $[\mathrm{BB}-\mathrm{O}]$, the integrability condition of the almost complex structures for G-structures of order 2 is given by the vanishing of a component of the Weyl tensor. In this paper we treat the case when

$$
\mathbf{R}^{2 n}=\mathbf{R}^{4}, G=S O_{+}(3,1), G_{0}=S O(3), C \cong S^{2} .
$$

The choice of the geometrical $T$ in this paper satisfies the assumption of this proposition. In this case, we express the integrability condition by the vanishing of certain components of the curvature tensor.

\section{Remark C:}

1. If $N^{3}$ is a 3 -dimensional oriented Riemannian manifold with constant curvature, the unit tangent bundle $T_{1}\left(N^{3}\right)$ of $N^{3}$ admits a normal CR-structure (cf. $[\mathrm{S}-\mathrm{Y}]$ ). Then we can naturally define the complex structure on $T_{1}\left(N^{3}\right) \times \mathbf{R}$. As $T_{1}\left(N^{3}\right) \cong N^{3} \times S^{2}$, we have

$$
T_{1}\left(N^{3}\right) \times \mathbf{R} \cong\left(N^{3} \times S^{2}\right) \times \mathbf{R} \cong\left(N^{3} \times \mathbf{R}\right) \times S^{2} .
$$

Put $M^{4}=N^{3} \times \mathbf{R}$. Then $M^{4}$ with the canonical Riemannian product metric is conformally flat, and self-dual. The twistor space of $M^{4}$ constructed by Atiyah-Hitchin-Singer is diffeomorphic to $M^{4} \times S^{2}=\left(N^{3} \times \mathbf{R}\right) \times S^{2}$. Its complex structure coincides with the above one. On the other hand, the space-time $M^{4}$ with the canonical Lorentzian product metric is conformally flat, too. The complex manifold $M^{4} \times S^{2}$ which is complex 3 -dimensional can be regarded as the space of all null directions. It arises a natural question when a complex structure is defined on the space of all null directions. We want to investigate its condition, properties, examples and classifications. This is another motivation of this paper.

2. Let $M$ be a 4-dimensional oriented and connected manifold. We restrict a discussion to a coordinate neighborhood locally. If we give a Lorentzian metric $g_{L}$ and a unit vector field $T\left(g_{L}(T, T)=-1\right)$ on $M$, a Riemannian metric $g_{R}(T)$ called Riemannization is canonically defined by changing norm -1 into norm 1 of $T$. If we give a Riemannian metric $g_{R}$ and a unit vector field $S\left(g_{R}(S, S)=1\right)$ on $M$, a Lorentzian metric $g_{L}(S)$ called Lorentzization is canonically defined by changing norm 1 into norm -1 of $S$.

For a Lorentzian metric $g_{L}$, the linear frame bundle over $M$ with structure group $G L(4, \mathbf{R})$ is reduced to a principal bundle $Q$ with structure group $S O(3,1)$. If we choose $T$ on $M$, then $Q$ is reduced to $Q_{T}$ with structure group 
$S O$ (3). We have the twistor space $P$ of $M$ given by

$$
P=Q \times_{S O(3,1)} S^{2}=Q_{T} \times_{S O(3)} S^{2} .
$$

The space $P$ is integrable if and only if the condition of Theorem in $\S 1$ is satisfied. For a Riemannian metric $g_{R}$, the linear frame bundle over $M$ with structure group $G L(4, \mathbf{R})$ is reduced to a principal bundle $F$ with structure group $S O(4)$. If we choose $S$ on $M$, then $F$ is reduced to $F_{S}$ with structure group $S O(3)$. The twistor space $Z$ of $M$ in [A-H-S] is given by

$$
Z=F \times_{\text {SO(4) }} S^{2}=F_{S} \times_{\text {SO(3) }} S^{2} .
$$

The space $Z$ is integrable if and only if $M$ is a self-dual manifold.

If we can take $T=S$, the twistor spaces $P$ and $Z$ coincide. But since the horizontal lift to $P$ is given by $S O(3,1)$-connection and the horizontal lift to $Z$ by $S O(4)$-connection, an almost complex structure $J_{T=S}$ on $P$ and an almost complex structure $J_{S=T}$ on $Z$ do not coincide in general. The twistor space of a conformally flat Lorentzian manifold is not necessary integrable, but the Riemannization is conformally flat and the twistor space is integrable.

Acknowledgement. The first named author would like to thank Professor Akihiro Tsuchiya for the advice on learning mathematical physics and for the encouragement during his stay in Nagoya University.

\section{REFERENCES}

[A-H-S] M.F. Atiyah, N.J. Hitchin and I.M. Singer, Self-duality in four-dimensional Riemannian geometry, Proc. R. S. Lond., A 362 (1978), 425-461.

[BB-O] L. Berard Bergery and T. Ochiai, On some generalization of the construction of twistor spaces, Global Riemannian geometry (T. J. Willmore and N.J. Hitchin, eds.), Ellis Horwood, Chichester, 1984, pp. 52-59.

[Be] A. L. Besse, Einstein manifolds, Springer-Verlag, Berlin-Heidelberg-New York, 1987.

[Fr] T. Friedrich, (ed.), Self-dual Riemannian geometry and instantons, Teubner, Leipzig, 1981.

[Ma] Yu. I. Manin, Mathematics and physics, Birkhäuser, Boston-Basel, 1981.

[O'N] B.O'Neill, Semi-Riemannian geometry, Academic Press, New York, 1983.

[P-W] R. Penrose and R.S. Ward, Twistors for flat and curved space-time, General relativity and gravitation vol. 2 (A. Held, ed.), Plenum Press, New YorkLondon, 1980, pp. 283-328.

[S-Y] H. Sato and K. Yamaguchi, Lie contact manifolds, Geometry of manifolds (K. Shiohama, ed.), Academic Press, New York, 1989, pp. 191-238.

[W-W] R. S. Ward and R.O. Wells Jr., Twistor geometry and field theory, Cambridge Univ. Press, Cambridge, 1990. 
Y. Machida

Numazu College of Technology 3600 Ooka

Numazu-shi, Shizuoka 410

Japan

H. Sato

Department of Mathematics

School of Science

Nagoya University

Chikusa-ku, Nagoya 464-01

Japan 